\title{
Retrolaminar versus epidural block for postoperative analgesia after minor video-assisted thoracic surgery: a retrospective, matched, non-inferiority study
}

\author{
Keiko Nobukuni ${ }^{1}$, Mariko Hatta ${ }^{1}$, Toshiaki Nakagaki ${ }^{1}$, Jun Yoshino ${ }^{1}$, Toshiro Obuchi ${ }^{2}$, Naoyuki Fujimura ${ }^{1 \wedge}$ \\ ${ }^{1}$ Department of Anesthesiology, St. Mary's Hospital, Our Lady of the Snow Social Medical Corporation, Kurume, Japan; ${ }^{2}$ Department of Thoracic \\ Surgery, St. Mary's Hospital, Our Lady of the Snow Social Medical Corporation, Kurume, Japan \\ Contributions: (I) Conception and design: N Fujimura, K Nobukuni; (II) Administrative support: N Fujimura, K Nobukuni; (III) Provision of study \\ materials or patients: T Obuchi, N Fujimura; (IV) Collection and assembly of data: All authors; (V) Data analysis and interpretation: All authors; (VI) \\ Manuscript writing: All authors; (VII) Final approval of manuscript: All authors. \\ Correspondence to: Naoyuki Fujimura, MD, PhD. Director, Central Surgical Center, Department of Anesthesiology, St. Mary's Hospital, Our Lady of \\ the Snow Social Medical Corporation, 422 Tsubukuhonmachi, Kurume 830-8543, Japan. Email: n-fujimura@st-mary-med.or.jp.
}

\begin{abstract}
Background: The role of thoracic epidural analgesia (TEA) for postoperative analgesia after video-assisted thoracic surgery (VATS) is still controversial. Some studies have reported the efficacy of ultrasound-guided retrolaminar block (RLB) for the postoperative management of pain after chest wall surgery. The purpose of this study was to compare the postoperative analgesic efficacy and adverse effects of ultrasound-guided RLB with those of TEA in patients undergoing minor VATS procedures.

Methods: A total of 192 relevant records of patients were enrolled in this study. We reviewed electronic medical records of patients undergoing minor VATS procedures under general anesthesia. The primary outcome was the median differences in the numerical rating scale (NRS) scores during rest between the groups at the morning of postoperative day 1 (POD $1 \mathrm{~m}$ ). A propensity-matched analysis incorporating preoperative variables was used to compare the efficacy of postoperative analgesia in two groups.

Results: Overall, 94 patients were identified for analysis. Propensity score matching resulted in 47 patients in each group. There were no significant differences in the NRS scores between the two groups. The median differences in NRS scores during rest between the two groups at POD $1 \mathrm{~m}$ were under 1, which indicates non-inferiority of RLB. There were no significant differences in the incidence of adverse effects and rescue dose of analgesic consumption between the two groups.

Conclusions: The analgesic effects of continuous ultrasound-guided RLB were non inferior to those of TEA for minor VATS procedures.
\end{abstract}

Keywords: Retrolaminar block (RLB); epidural analgesia; minor video-assisted thoracic surgery (VATS); postoperative analgesia

Submitted Feb 04, 2021. Accepted for publication Mar 14, 2021.

doi: $10.21037 /$ jtd-21-238

View this article at: http://dx.doi.org/10.21037/jtd-21-238

\section{Introduction}

Thoracic epidural analgesia (TEA) or thoracic paravertebral block (TPVB) have been used as gold standard analgesia for thoracic surgery (1-5). TEA and TPVB provide comparable pain relief after thoracic surgery $(4,6)$. TEA protect against pulmonary complications than intravenous opioid administration (2). However, TEA carries the risk of

\footnotetext{
$\wedge$ ORCID: 0000-0003-0937-6293.
} 
complications such as hypotension, epidural abscess, spinal hematoma and neurological injuries (1). TPVB reduces the risks of hypotension, urinary retention and other minor complications compared to TEA (5). However, TPVB has similar contraindications including coagulation disorders and anticoagulation $(3,7)$, and other major complications such as serious neurologic injuries same as TEA (3).

Video-assisted thoracic surgery (VATS) is a minimally invasive technique and has been widely performed with its advantages of reduced postoperative morbidity and early recovery. VATS reduces postoperative pain scores compared with that of open thoracotomy. However, VATS is still associated with moderate to severe postoperative pain requiring treatment $(8,9)$. Several studies described different analgesic technique for VATS. There is no consensus regarding optimal postoperative pain control after VATS. Although, the role of TEA and TPVB for postoperative analgesia after VATS is controversial (10), the majority still prefer TEA compared with other regional analgesia for the postoperative analgesia after VATS (11).

Minor VATS procedures, including VATS bullectomy and wedge resection for lung cancer, were performed using smaller incisions and leading to shorter hospital stay compared to those of VATS lobectomy (12). Although it is not known whether minor VATS procedures are associated with less perioperative pain scores compared with that of VATS lobectomy, it should have less-invasive analgesia with less complications.

Ultrasound-guided retrolaminar block (RLB) $(13,14)$ are new thoracic truncal blocks. RLB are considered to be easy, less invasive and safe maneuvers compared to TEA, erector spinae plane block (ESPB) and TPVB (15). Although RLB can be performed without ultrasonography, ultrasound images allow for visualization of the needle, catheter and local anesthetic distribution. Some studies reported the application of RLB for chest wall surgery (16-19). RLB provided effective analgesia in cases of multiple rib fractures (13). Continuous infusion of local anesthetics through catheter prolongs the analgesic effects. However, there were no clinical reports that compare the analgesic efficacy of continuous RLB with that of TEA after thoracic surgery. We hypothesized that ultrasound-guided continuous RLB might provide analgesia as effective as that of TEA for minor VATS procedures. Thus, the purpose of this retrospective propensity-matched study was to compare the postoperative analgesic efficacy and adverse effects of ultrasound-guided continuous RLB with those of TEA in patients undergoing minor VATS procedures.
We present the following article in accordance with the STROBE reporting checklist (available at http://dx.doi. org/10.21037/jtd-21-238).

\section{Methods}

\section{Ethics, study participant enrollment}

This study was conducted in accordance with the Declaration of Helsinki (as revised in 2013). This study protocol was examined and approved by the Research Review Board at St. Mary's Hospital on 12 January, 2017 (Approval No., \#16-1205). Written informed consent was obtained from all patients who participated in this study.

We enrolled the patients with American Society of Anesthesiologists physical status (ASA-PS) 1-2 who were scheduled for minor VATS procedures (bullectomy, partial lung resection and mediastinal tumor resection) at St. Mary's Hospital from February 2017 to December 2019. Exclusion criteria included patients with bilateral pneumothorax, bilateral thoracic surgery, redo surgery, chronic pain, preoperative use of opioid or pain medication, history of substance use, alcohol abuse, ASA-PS 3 or greater, younger than 15 years old, coagulation abnormalities (platelet count less than $100 \times 10^{3} / \mu \mathrm{L}$, prothrombin time-international normalized ratio (PT-INR) greater than 1.2 or activated partial thromboplastin time (APTT) longer than normal range), allergy to any drug used in this study, and inability to provide informed consent.

\section{Ultrasound-guided RLB and TEA}

Ultrasound-guided RLB catheterization was performed under general anesthesia after the end of surgery in the lateral position using a linear ultrasound probe (15-6 MHz; S-Nerve ${ }^{\mathrm{TM}}$, FUJIFILM SonoSite, Bothell, Washington).

The RLB was performed as previously described (13). In the RLB group, a 17-G Tuohy epidural needle (Perican ${ }^{\circledR}$; B-Braun Medical, Melsungen GA, Germany) was inserted using in-plane ultrasound view, from cephalad to caudal direction at $1 \mathrm{~cm}$ lateral to the spinous process at $\mathrm{T} 4$ or T5, where the main surgical incision was made. After the confirmation hydro-dissection (normal saline) of the interfascial plane, the mixture of ropivacaine $0.75 \%$ $10 \mathrm{~mL}$ plus lidocaine $1 \%$ with $1: 100,000$ epinephrine $10 \mathrm{~mL}$ were administered when the needle tip reached the lamina. After the placement of the catheter (FX catheter ${ }^{\circledR}$; B-Braun Medical, Melsungen GA, Germany) $5.0 \mathrm{~cm}$ 
beyond the needle tip, ropivacaine $0.2 \%(8-12 \mathrm{~mL} / \mathrm{h})$ was continuously administered using a balloon injector (Rakuraku fuser ambulatory disposable pump ${ }^{\circledR}$, Smiths Medical, Minneapolis, MN, USA) until the chest tube was removed.

The epidural catheter was placed without sedation before induction of general anesthesia via a paramedian approach in the lateral decubitus position. After local anesthesia with $1 \%$ lidocaine, a Tuohy $17-\mathrm{G}$ epidural needle was inserted at T4/5 or T5/6 intervertebral space. Epidural space was identified by the loss of resistance technique. The catheter was advanced 4 to $5 \mathrm{~cm}$ into the epidural space. After a test aspiration was negative, a $3-\mathrm{mL}$ test dose of $1 \%$ lidocaine was administered. Postoperative analgesia was initiated with a single shot administration of ropivacaine $0.375 \%(3-6 \mathrm{~mL})$ at the end of surgery. Ropivacaine $0.2 \%$ (3-6 $\mathrm{mL} / \mathrm{h}$ ) was continuously administered until the chest tube was removed.

The responsible anesthesiologists decided the choice of analgesia and dose for continuous infusion of local anesthetics. Patient controlled regional analgesia was not performed in this study. If adverse events such as postoperative nausea and vomiting (PONV) occurred, the local anesthetic flow was stopped or decreased until the event subsided, and then the flow was resumed. The decision was made by the anesthesiologists or surgeons.

\section{General anesthesia}

Premedication and pre-operative multi-modal pain medication were not given to the patients. A standard monitor was attached to the patient after entering the operating room. General anesthesia was induced with propofol $(1-2 \mathrm{mg} / \mathrm{kg})$, fentanyl $(50 \mu \mathrm{g})$ and rocuronium $(0.6 \mathrm{mg} / \mathrm{kg})$. A left-sided double-lumen endobronchial tube (Shiley ${ }^{\mathrm{TM}}$, Covidien, Mansfield, MA, USA) was placed for one lung ventilation, and its correct position was confirmed by auscultation and fiber-optic bronchoscopy. The maintenance of anesthesia consisted of balanced anesthesia of inhalational anesthesia (sevoflurane, desflurane) with remifentanil, or propofol with remifentanil. Intravenous fentanyl and rocuronium were administered as needed. Acetaminophen or flurbiprofen were administered intravenously at skin closure. Administration of inhalational anesthesia, propofol and remifentanil were discontinued upon completion of the surgery, and sugammadex was administered to reverse the muscle relaxation induced by rocuronium. Fentanyl $(50 \mu \mathrm{g})$ or pethidine $(10 \mathrm{mg})$ was administered incrementally if there was request of rescue analgesia after awaking from anesthesia. The patient was discharged from the operating theater and sent to the ward after achieving an appropriate level of consciousness and analgesia. Narcotic rescue usage is standardized to morphine milligram equivalent (MME; morphine:pethidine:fentanyl $=1: 10: 0.01$ ) (20). Narcotics were only available for patients in the operating room.

\section{Postoperative pain management}

In our institute, preoperative multimodal pain management was not performed. Regular postoperative analgesics consisted of loxoprofen sodium hydrate $(60 \mathrm{mg})$ three times daily. Intravenous flurbiprofen or acetaminophen were used for rescue analgesia when the pain was not well controlled (NRS $\geq 5$ or request of rescue analgesia) despite TEA or RLB uses.

The postoperative pain intensity was assessed by the numerical rating scale (NRS) scores at 6 and $12 \mathrm{~h}$ after surgery, the morning and evening of postoperative day (POD) 1 and the morning of POD 2. NRS scores were recorded at rest (during quiet breathing) and when coughing.

\section{Surgical procedures}

In our institute, a three-port technique was used for VATS port-site strategy. The 6th to 8 th intercostal spaces were used for the placement of the inferior port for videoscope access and chest tube placement. The 4th to 5 th intercostal spaces were used for the placement of the anterior and posterior ports. The incision site was limited between the 4th and 8 th intercostal spaces. All surgeries were performed by the same operation team.

\section{Outcomes}

The primary outcome of this study was the median differences in the NRS scores between the TEA and RLB groups during rest at the morning of postoperative day 1 (POD $1 \mathrm{~m}$ ). The secondary outcome parameters were NRS scores at rest and when coughing, postoperative initial narcotic rescue usage, rescue analgesics requirement in the ward, and adverse effects including incidence of hypotension, pruritus, urinary retention, PONV, and the length of postoperative hospitalization. In routine practice, the nursing staff asked the patients about pruritus, urinary 
retention, nausea and pain intensity. NRS scores were recorded 6 and $12 \mathrm{~h}$ after surgery, POD $1 \mathrm{~m}$, the evening of POD 1 (POD 1e) and the morning of POD 2 (POD 2m). Hypotension was defined as systolic blood pressure less than $90 \mathrm{mmHg}$.

\section{Propensity-matched design}

Patients who received TEA were matched to those who received RLB at a ratio using propensity score matching at a ratio of 1:1. This matching was used to obtain groups of patients corresponding to the 2 analgesic methods that were balanced with regard to potential confounding baseline variables. To estimate the propensity score, a multivariable logistic regression analysis was used based on the following covariates: age, sex, height, body weight, ASA class, surgical procedures, and procedural duration of surgery. We did not include the surgeon as a confounder, because the same operation team conducted all our surgeries.

Propensity matching was performed by the nearestneighbor algorithm without replacement and patients who received TEA (TEA group) and RLB (RLB group) were matched by a 1:1 ratio. This matching was performed using a caliper width of 0.2 standard deviations of the logit of the estimated propensity score. The standardized difference was estimated to evaluate covariate balance, whereby an absolute standardized difference above 0.1 represents meaningful imbalance.

\section{Statistical analysis}

Data are expressed as median [interquartile range (IQR)] and numbers with percentage. Statistical analysis was performed using, $\chi^{2}$ test, Fisher's exact test and MannWhitney $\mathrm{U}$ test. The level of significance was set at $\mathrm{P}$ value $<0.05$. Statistical analyses were performed with EZR (Saitama Medical Center, Jichi Medical University, Saitama, Japan), which is a graphical user interface for $\mathrm{R}$ (The $\mathrm{R}$ Foundation for Statistical Computing, Vienna, Austria). More precisely, it is a modified version of $\mathrm{R}$ commander designed to add statistical functions frequently used in biostatistics.

We calculated the $95 \%$ CI of the median differences in NRS scores using the Hodges-Lehmann estimator. We defined an acceptable non-inferiority margin as 1.0 according to the previous study (21). For the non-inferiority evaluation, we used the R statistical package (version 3.5.2, R Foundation for Statistical Computing).

\section{Results}

We reviewed electronic medical records of patients undergoing VATS under general anesthesia from February 1, 2017 to December 31, 2019. A total of 438 relevant records of patients were reviewed in this study. Of these, 344 records were excluded (Figure 1), and 94 patients were successfully matched on a 1:1 basis, based on predetermined confounders and baseline characteristics, including 47 patients in each of the RLB and the TEA groups. Table 1 summarizes patient baseline characteristics after propensity score matching. Postoperative NRS scores between the TEA group and the RLB group before propensity score matching is available in the Supplemental material (Table S1). Patients in the matched population were similar with respect to matched baseline characteristics.

\section{Patient demographics}

There were no significant differences in the type of surgery and the duration of operation between the two groups. The duration of general anesthesia was significantly longer in the RLB group than in the TEA group [124.0 (112.5133.0) $\mathrm{min}$ in the RLB group and 96.0 (85.0-111.0) $\mathrm{min}$ in the TEA group $(\mathrm{P}<0.01)]$. Intraoperative remifentanil consumption was significantly higher in the RLB group than in the TEA group [1.3 (1.1-1.7) $\mathrm{mg}$ in the RLB group and $1.0(0.7-1.3) \mathrm{mg}$ in the TEA group $(\mathrm{P}<0.01)]$. There were no significant differences in the intraoperative fentanyl consumption between the two groups [200.0 (100.0-250.0) $\mu \mathrm{g}$ in the RLB group and 150.0 (100.0-200.0) $\mu \mathrm{g}$ in the TEA group $(\mathrm{P}=0.13)]$. There were no significant differences in the duration of postoperative thoracic drainage between the two groups [2.0 (2.0-4.0) days in the RLB group and $2.0(2.0-3.5)$ days in the TEA group $(\mathrm{P}=0.345)]$. There were no significant differences in the length of postoperative hospitalization between the two groups [6.0 (5.0-8.5) days in the RLB group and 5.0 (4.0-8.0) days in the TEA group $(\mathrm{P}=0.226)]$.

\section{Analgesic efficacy}

Postoperative NRS scores and median differences for NRS scores between the two group at rest and when coughing are shown in Table 2. There were no significant differences in the NRS scores at rest and when coughing between the two groups at 6 and $12 \mathrm{~h}$ after surgery, POD $1 \mathrm{~m}$, POD $1 \mathrm{e}$ and POD $2 \mathrm{~m}$. 


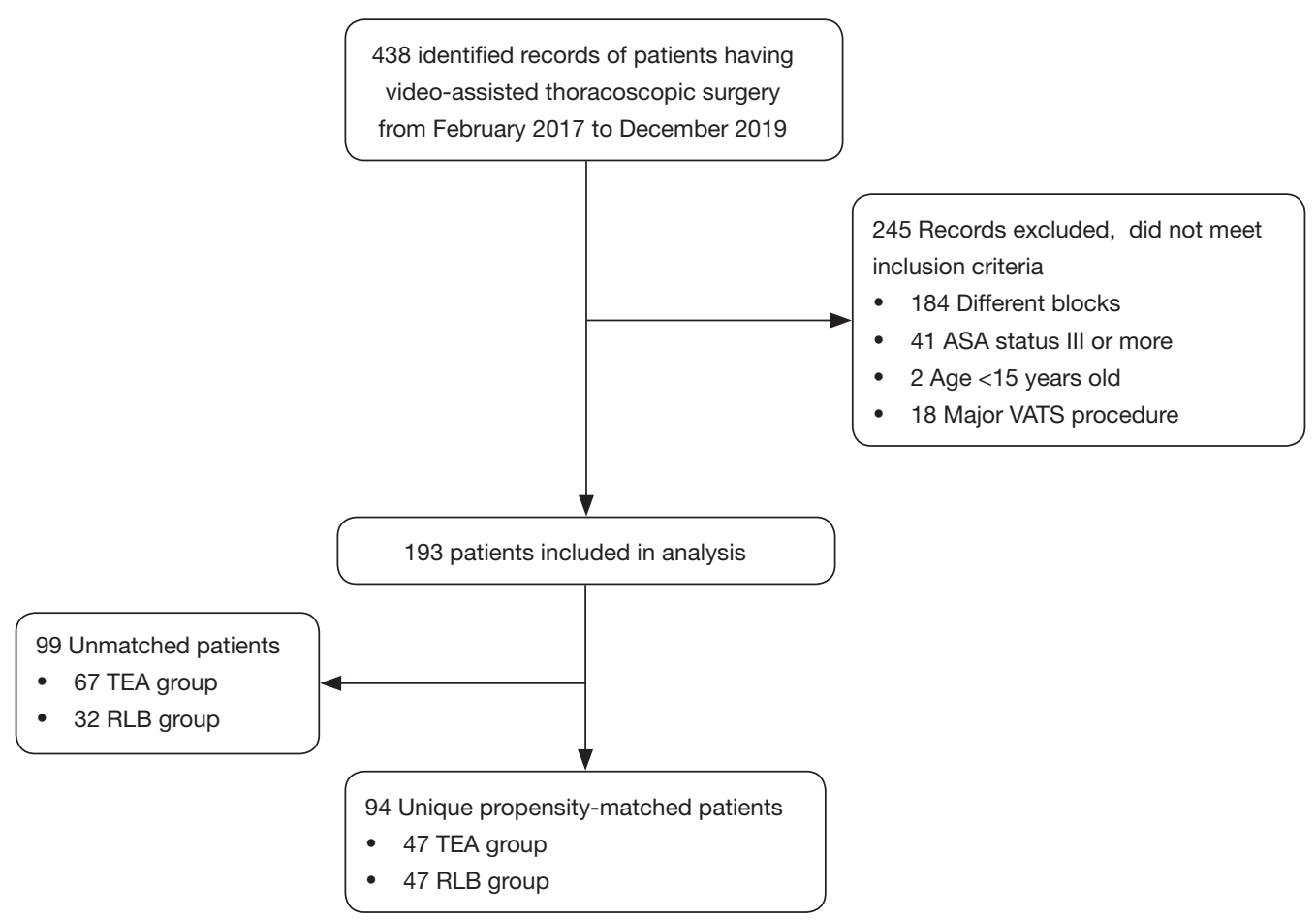

Figure 1 Flow diagram of case selection. VATS, video-assisted thoracic surgery; TEA, thoracic epidural analgesia; RLB, retrolaminar block; ASA, American Society of Anesthesiologists.

Table 1 Patient characteristics after propensity score matching

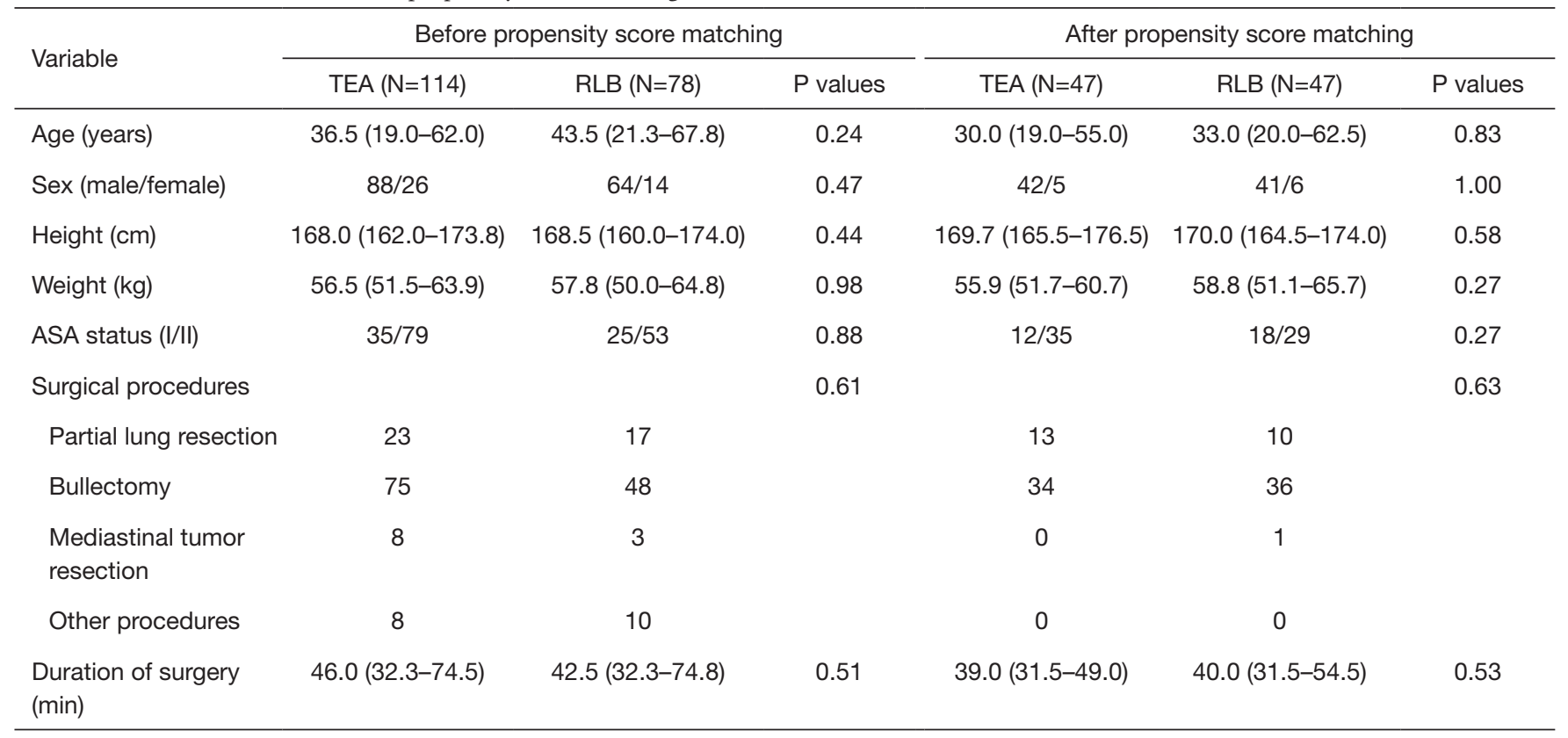

Data are presented as median (IQR) or absolute numbers. $\chi^{2}$ test, Fisher's exact test or Mann-Whitney $U$ test was used as appropriate for the distribution as the distributions would affect whether parametric or non-parametric testing is used. ASA, American Society of Anesthesiologists; TEA, thoracic epidural analgesia; RLB, retrolaminar block; IQR, interquartile range. 
Table 2 Postoperative numerical rating scale (NRS) scores and median differences for NRS scores between the TEA group and the RLB group

\begin{tabular}{|c|c|c|c|c|}
\hline Time & TEA $(\mathrm{N}=47)$ & RLB (N=47) & $P$ value (TEA vs. RLB) & Differences in NRS (RLB - TEA) \\
\hline $6 \mathrm{~h}$ & $1.0(0.0-3.0)$ & $2.5(0.0-4.0)$ & 0.31 & $0.0(0.0,1.0)$ \\
\hline $12 \mathrm{~h}$ & $1.0(0.0-3.0)$ & $2.0(0.0-4.0)$ & 0.56 & $0.0(0.0,1.0)$ \\
\hline POD 1m & $2.0(0.0-3.0)$ & $2.0(0.0-3.0)$ & 0.89 & $0.0(-1.0,1.0)$ \\
\hline POD 2m & $1.0(0.0-3.0)$ & $1.0(0.0-3.0)$ & 0.75 & $0.0(-1.0,0.0)$ \\
\hline \multicolumn{5}{|c|}{ NRS when moving } \\
\hline $6 \mathrm{~h}$ & $3.0(0.0-5.0)$ & $4.0(2.5-5.0)$ & 0.24 & $1.0(0.0,2.0)$ \\
\hline $12 \mathrm{~h}$ & $3.0(1.0-6.0)$ & $3.0(2.5-5.0)$ & 0.76 & $0.0(-1.0,1.0)$ \\
\hline POD 2m & $3.0(2.0-4.0)$ & $3.0(2.0-4.0)$ & 0.67 & $0.0(-1.0,1.0)$ \\
\hline
\end{tabular}

Data are presented as median (IQR). NRS scores were analyzed using the Mann-Whitney $\mathrm{U}$ test. Hodges-Lehman estimator was used to calculate $95 \% \mathrm{Cl}$ of the median differences. NRS; numerical rating scale; TEA, thoracic epidural analgesia; RLB, retrolaminar block; 6 h, 6 hours after surgery; 12 h, 12 hours after surgery; POD 1m, the morning of postoperative day 1; POD 1e, the evening of postoperative day 1 ; POD $2 \mathrm{~m}$, the morning of postoperative day 2 .

The $95 \%$ CI of the median differences in NRS scores between the two groups are shown in Figure 2. The 95\% CI of the median differences in NRS scores during rest between the two groups at POD $1 \mathrm{~m}$ were under 1 , which indicates non-inferiority of RLB. The $95 \%$ CI of the median differences in NRS scores when coughing between the two groups except for $6 \mathrm{~h}$ after surgery were under 1 . The $95 \%$ CI of the median differences in NRS scores when coughing at $6 \mathrm{~h}$ after surgery was over 1 .

\section{Analgesic consumption and adverse effects after surgery}

There were no significant differences in the narcotic rescue usage (MME) in the operating room between the two groups $[0.0(0.0-0.0)$ vs. $0.0(0.0-0.0) \mathrm{mg}$, respectively, $\mathrm{P}=0.1]$.

The initial infusion dose of local anesthetics was significantly higher in the RLB group than in the TEA group $[10.0(9.0-10.0) \mathrm{mL} / \mathrm{h}$ in the RLB group and 5.0 $(4.0-5.0) \mathrm{mL} / \mathrm{h}$ in the TEA group $(\mathrm{P}<0.01)]$. The infusion dose of local anesthetics at POD $1 \mathrm{~m}$ were significantly higher in the RLB group than in the TEA group [8.0 $(6.0-10.0) \mathrm{mL} / \mathrm{h}$ in the RLB group and $4.0(3.0-5.0) \mathrm{mL} / \mathrm{h}$ in the TEA group $(\mathrm{P}<0.01)$.

Table 3 shows frequency of rescue analgesics use and incidence of PONV after surgery in the ward. There were no significant differences in the frequency of rescue analgesics use between the two groups $(\mathrm{P}=0.87)$. There were no significant differences in the incidence of $\mathrm{PONV}$ between the two groups $(\mathrm{P}=0.17)$. There were no patients who developed hypotension between the two groups. There was no occurrence of pruritus or urinary retention between the two groups.

\section{Discussion}

This retrospective study compared the postoperative analgesic efficacy and adverse effects of ultrasound-guided RLB with those of TEA in patients undergoing minor VATS procedures. The analgesic effects of RLB after minor VATS procedures were similarly effective in reducing postoperative pain compared to those of TEA except for $6 \mathrm{~h}$ after surgery when coughing. There were no significant differences in the incidence of adverse effects or rescue doses of analgesics between the two groups. These results suggested that continuous RLB provided effective analgesia comparable to that of TEA for minor VATS procedures.

The analgesic effects of thoracic wall blocks is dependent on passive spread of local anesthetics. Local anesthetics injected into a tissue plane passively spread within the 
A

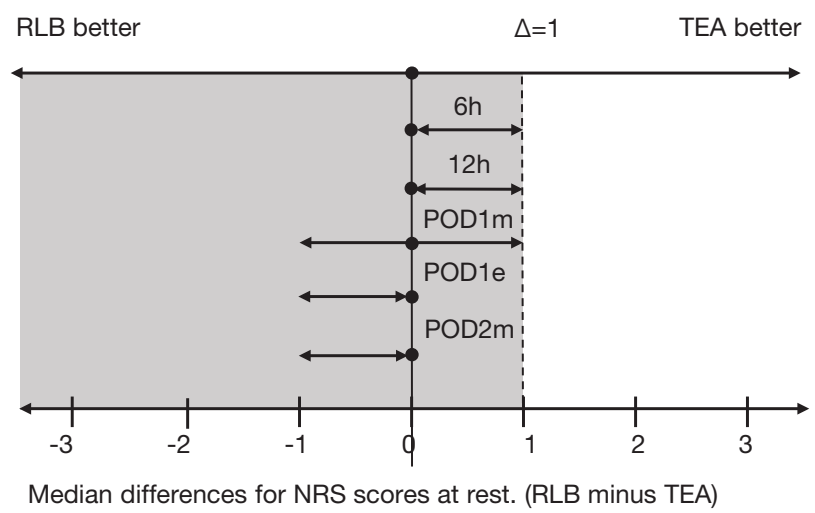

B

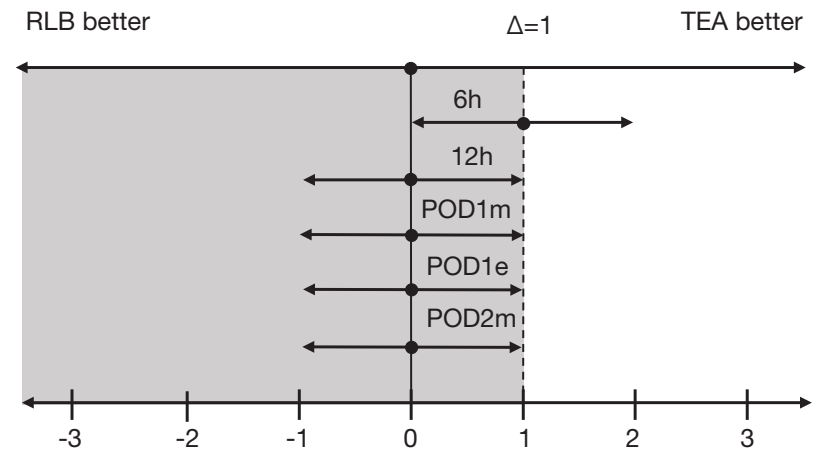

Median differences for NRS scores when moving. (RLB minus TEA)

Figure 2 Median differences for NRS scores (RLB minus TEA). (A) Median differences for NRS scores at rest (RLB minus TEA); (B) median differences for NRS scores when coughing (RLB minus TEA). TEA, thoracic epidural analgesia; RLB, retrolaminar block; 6 h, 6 hours after surgery; 12 h, 12 hours after surgery; POD 1m, the morning of postoperative day 1; POD 1e, the evening of postoperative day 1 ; POD $2 \mathrm{~m}$, the morning of postoperative day 2.

Table 3 Number of postoperative analgesics used and incidence of PONV

\begin{tabular}{lccc}
\hline Variables & TEA (N=47) & RLB (N=47) & P value \\
\hline $\begin{array}{l}\text { Number of additional } \\
\text { analgesics use, } \mathrm{n}\end{array}$ & & & 0.87 \\
0 time & 14 & 30 & \\
1 time & 5 & 11 & \\
2 times & 1 & 1 & \\
3 times & 0 & 0 & \\
4 time & 1 & 0 & 0.17 \\
5 time & $17(36.2)$ & $10(21.3)$ & \\
Incidence of PONV, $\mathrm{n}(\%)$ & 14 & & \\
\hline
\end{tabular}

Data are presented as absolute numbers (\%). P compares the TEA and the RLB groups. Number of additional analgesics used and postoperative incidence of PONV were analyzed using Fisher's exact test. PONV, postoperative nausea and vomiting; TEA, thoracic epidural analgesia; RLB, retrolaminar block.

tissue plane or to adjacent tissue compartments containing nerves. Total injected mass of local anesthetics that reach and act on target nerves exert analgesia (22). Damjanovska et al. (23) reported that the spread of injectate from retrolaminar to paravertebral space is volume dependent in porcine cadaver models. Local anesthetics administered as an initial bolus dose of $20 \mathrm{~mL}$, followed by continuous infusion of $8-12 \mathrm{~mL} / \mathrm{h}$ in our study. Although our dosage used in this study provided effective analgesia comparable to that of TEA for minor VATS procedures, the optimal dosage of local anesthetic required for achieving effective analgesia is still not clear.

RLB are the alternatives to TEA and TPVB. Local anesthetics are injected into the fascial plane between the posterior surface of the lamina and the overlying transversospinalis muscles at $1-1.5 \mathrm{~cm}$ lateral to the spinous process in RLB (22). It was considered that local anesthetics penetrate the superior costotransverse ligament and spread to the thoracic paravertebral space (24-26). Sabouri et al. demonstrated that the dye injected at the retrolaminar space spread into paravertebral space, epidural space and intervertebral foramina (25). Adhikary et al. reported injectate spread to the epidural and neural foraminal spaces over 2 to 5 levels (26), and confirmed the existence of anatomical pathways for anterior spread of local anesthetics. Due to the nature of the cadaveric studies, it is hard to clarify the exact mechanisms of RLB. Furthermore, the determinant of the clinical efficacy of RLB in actual patients is still not clear.

In our study, the duration of general anesthesia was significantly longer in the RLB groups than in the TEA group. The longer duration of anesthesia was accompanied with higher intraoperative remifentanil consumption in the RLB group compared to that of TEA group. There were no significant differences in the duration of surgery. The epidural catheter was placed without sedation before induction of general anesthesia, on the other hand, RLB catheterization was performed under general anesthesia 
after the end of surgery. The methodological differences of catheterization might be responsible for the differences of duration of anesthesia and intraoperative remifentanil consumption between the two groups.

RLB can be performed easily and safely under ultrasound guidance. The fourth edition of the American Society of Regional Anesthesia and Pain Medicine Evidence-Based Guidelines (7) suggested that for procedures other than peri-neuraxial, deep plexus, or deep peripheral blocks, management should be based on the consideration of site compressibility, vascularity and consequence of bleeding. The RLB injects local anesthetics between the thoracic laminae and the deep paraspinous muscles. There are no major vessels at the site of injection. The site of injection of RLB are compressible because of the superficiality of the injection site and bony floor. Theoretically RLB could reduce the risk of epidural hematoma, spinal cord and nerve injury which are considered as a serious complication of TEA. RLB could also reduce the risk of pneumothorax and incidental epidural injection of local anesthetics considered as a complication of TPVB (15). RLB are considered to be safer maneuvers than TEA and TPVB.

There are several limitations in this study that include those inherent to retrospective study. First, this is a singlecenter study with small sample size. The population examined in this study may not be representative of the general population. Second, different individual anesthetist decided the method of analgesia. Propensity score matching analysis may have reduced the risk of bias and improved the validity of our analysis; selection bias was not eliminated. Third, we did not assess the dermatomal distribution after either block. Furthermore, different doses between the RLB and TEA may cause bias the result. Fourth, our database was insufficient to allow us to comment on outcomes such as PONV intensity. Fifth, the NRS scores were recorded by the nursing staffs, it might bias the results by which patient would overrate their pain in order to ensure rescue medications administered by the same nursing team.

However, despite the limitations, we hope to improve this observation in prospective clinical trials.

\section{Conclusions}

Our study suggested that the analgesic effects of continuous ultrasound-guided RLB were non-inferior to those of TEA for minor VATS procedures.

\section{Acknowledgments}

We appreciate the contribution of the colleagues who participated in the research.

Funding: None.

\section{Footnote}

Reporting Checklist: The authors have completed the STROBE reporting checklist. Available at http://dx.doi. org/10.21037/jtd-21-238

Data Sharing Statement: Available at http://dx.doi. org/10.21037/jtd-21-238

Peer Review File: Available at http://dx.doi.org/10.21037/jtd21-238

Conflicts of Interest: All authors have completed the ICMJE uniform disclosure form (available at http://dx.doi. org/10.21037/jtd-21-238). The authors have no conflicts of interest to declare.

Ethical Statement: The authors are accountable for all aspects of the work in ensuring that questions related to the accuracy or integrity of any part of the work are appropriately investigated and resolved. This study was conducted in accordance with the Declaration of Helsinki (as revised in 2013). This study protocol was examined and approved by the Research Review Board at St. Mary's Hospital on 12 January 2017 (Approval No, \#16-1205). Written informed consent was obtained from all patients who participated in this study.

Open Access Statement: This is an Open Access article distributed in accordance with the Creative Commons Attribution-NonCommercial-NoDerivs 4.0 International License (CC BY-NC-ND 4.0), which permits the noncommercial replication and distribution of the article with the strict proviso that no changes or edits are made and the original work is properly cited (including links to both the formal publication through the relevant DOI and the license). See: https://creativecommons.org/licenses/by-nc-nd/4.0/. 


\section{References}

1. Freise H, Van Aken HK. Risks and benefits of thoracic epidural anaesthesia. Br J Anaesth 2011;107:859-68.

2. Pöpping DM, Elia N, Marret E, et al. Protective effects of epidural analgesia on pulmonary complications after abdominal and thoracic surgery: A meta-analysis. Arch Surg 2008;143:990-9.

3. D'Ercole F, Arora H, Kumar PA. Paravertebral Block for Thoracic Surgery. J Cardiothorac Vasc Anesth 2018;32:915-27.

4. Scarfe AJ, Schuhmann-Hingel S, Duncan JK, et al. Continuous paravertebral block for post-cardiothoracic surgery analgesia: A systematic review and meta-analysis. Eur J Cardiothorac Surg 2016;50:1010-8.

5. Yeung JH, Gates S, Naidu BV, et al. Paravertebral block versus thoracic epidural for patients undergoing thoracotomy. Cochrane Database Syst Rev 2016;2:CD009121.

6. Davies RG, Myles PS, Graham JM. A comparison of the analgesic efficacy and side-effects of paravertebral vs epidural blockade for thoracotomy - A systematic review and meta-analysis of randomized trials. Br J Anaesth 2006;96:418-26.

7. Horlocker TT, Vandermeuelen E, Kopp SL, et al. Regional Anesthesia in the Patient Receiving Antithrombotic or Thrombolytic Therapy: American Society of Regional Anesthesia and Pain Medicine Evidence-Based Guidelines (Fourth Edition). Reg Anesth Pain Med 2018;43:263-309.

8. Obuchi T, Yoshida Y, Moroga T, et al. Postoperative pain in thoracic surgery: Re-evaluating the benefits of VATS when coupled with epidural analgesia. J Thorac Dis 2017;9:4347-52.

9. Steinthorsdottir KJ, Wildgaard L, Hansen HJ, et al. Regional analgesia for video-assisted thoracic surgery: A systematic review. Eur J Cardiothorac Surg 2014;45:959-66.

10. Sondekoppam RV, Tsui BCH. "Minimally invasive" regional anesthesia and the expanding use of interfascial plane blocks: the need for more systematic evaluation. Can J Anaesth 2019;66:855-63.

11. Shanthanna H, Moisuik P, O'Hare T, et al. Survey of Postoperative Regional Analgesia for Thoracoscopic Surgeries in Canada. J Cardiothorac Vasc Anesth 2018;32:1750-5.

12. von Meyenfeldt EM, Marres GMH, van Thiel E, et al. Variation in length of hospital stay after lung cancer surgery in the Netherlands. Eur J Cardiothorac Surg 2018;54:560-4.
13. Voscopoulos C, Palaniappan D, Zeballos J, et al. The ultrasound-guided retrolaminar block. Can J Anaesth 2013;60:888-95.

14. Zeballos JL, Voscopoulos C, Kapottos M, et al. Ultrasound-guided retrolaminar paravertebral block. Anaesthesia 2013;68:649-51.

15. Onishi E, Toda N, Kameyama Y, et al. Comparison of Clinical Efficacy and Anatomical Investigation between Retrolaminar Block and Erector Spinae Plane Block. Biomed Res Int 2019;2019:2578396.

16. Murouchi T, Yamakage M. Retrolaminar block: analgesic efficacy and safety evaluation. J Anesth 2016;30:1003-7.

17. Onishi E, Murakami M, Nishino R, et al. Analgesic effect of double-level retrolaminar paravertebral block for breast cancer surgery in the early postoperative period: A placebo-controlled, randomized clinical trial. Tohoku J Exp Med 2018;245:179-85.

18. Hwang BY, Kim E, Kwon JY, et al. The analgesic efficacy of a single injection of ultrasound-guided retrolaminar paravertebral block for breast surgery: A prospective, randomized, double-blinded study. Korean J Pain 2020;33:378-85.

19. Sotome S, Sawada A, Wada A, et al. Erector spinae plane block versus retrolaminar block for postoperative analgesia after breast surgery: a randomized controlled trial. J Anesth 2021;35:27-34.

20. Woodhouse A, Hobbes AFT, Mather LE, et al. A comparison of morphine, pethidine and fentanyl in the postsurgical patient-controlled analgesia environment. Pain 1996;64:115-21.

21. Taketa Y, Irisawa Y, Fujitani T. Comparison of ultrasound-guided erector spinae plane block and thoracic paravertebral block for postoperative analgesia after video-assisted thoracic surgery: a randomized controlled non-inferiority clinical trial. Reg Anesth Pain Med 2019;45:10-5.

22. Chin KJ. Thoracic wall blocks: From paravertebral to retrolaminar to serratus to erector spinae and back again - A review of evidence. Best Pract Res Clin Anaesthesiol 2019;33:67-77.

23. Damjanovska M, Pintaric TS, Cvetko E, et al. The ultrasound-guided retrolaminar block: Volume-dependent injectate distribution. J Pain Res 2018;11:293-9.

24. Costache I, Sinclair J, Farrash FA, et al. Does paravertebral block require access to the paravertebral space? Anaesthesia 2016;71:858-9.

25. Sabouri AS, Crawford L, Bick SK, et al. Is a Retrolaminar Approach to the Thoracic Paravertebral Space Possible?: 
A Human Cadaveric Study. Reg Anesth Pain Med 2018;43:864-8.

26. Adhikary SD, Bernard S, Lopez H, et al. Erector Spinae
Plane Block Versus Retrolaminar Block: A Magnetic Resonance Imaging and Anatomical Study. Reg Anesth Pain Med 2018;43:756-62.

Cite this article as: Nobukuni $\mathrm{K}$, Hatta $\mathrm{M}$, Nakagaki T, Yoshino J, Obuchi T, Fujimura N. Retrolaminar versus epidural block for postoperative analgesia after minor video-assisted thoracic surgery: a retrospective, matched, non-inferiority study. J Thorac Dis 2021;13(5):2758-2767. doi: 10.21037/jtd-21238 
Supplementary

Table S1 Postoperative numerical rating scale (NRS) scores between the TEA group and the RLB group before propensity score matching

\begin{tabular}{llll}
\hline Time & TEA $(\mathrm{N}=114)$ & RLB $(\mathrm{N}=78)$ & P value (TEA vs. RLB) \\
\hline NRS at rest & & & \\
$6 \mathrm{~h}$ & $1.0(0.0-3.0)$ & $2.5(0.0-4.0)$ & 0.24 \\
$12 \mathrm{~h}$ & $1.0(0.0-3.0)$ & $2.0(0.0-3.0)$ & 0.40 \\
POD 1m & $2.0(0.0-3.0)$ & $2.0(0.0-3.0)$ & 0.30 \\
POD 1e & $2.0(0.0-3.0)$ & $2.0(0.0-3.0)$ & 0.61 \\
POD 2m & $1.0(0.0-3.0)$ & $1.0(0.0-3.0)$ & 0.52 \\
NRS when moving & & & 0.98 \\
6 h & $3.0(1.0-5.0)$ & $3.0(1.0-5.0)$ & 0.87 \\
$12 \mathrm{~h}$ & $3.0(1.0-5.0)$ & $3.0(2.0-5.0)$ & 0.61 \\
POD 1m & $3.0(2.0-5.0)$ & $3.0(1.0-5.3)$ & 0.96 \\
POD 1e & $3.5(2.0-5.0)$ & $4.0(1.0-5.0)$ & $3.0(1.0-4.0)$ \\
POD 2m & $3.0(2.0-4.3)$ & & \\
\hline
\end{tabular}

Data are presented as median (IQR). NRS scores were analyzed using the Mann-Whitney $U$ test. NRS, numerical rating scale; TEA, thoracic epidural analgesia; RLB, retrolaminar block; 6 h, 6 hours after surgery; 12 h, 12 hours after surgery; POD 1m, the morning of postoperative day 1; POD 1e, the evening of postoperative day 1 ; POD $2 \mathrm{~m}$, the morning of postoperative day 2. 